\title{
The feasibility and safety of repeat cryosurgical ablation of localized prostate cancer
}

\author{
Mahmoud Mustafa ${ }^{1,2^{*}}$, Scott Delacroix ${ }^{2}$, John F. Ward ${ }^{2}$ and Louis Pisters ${ }^{2}$
}

\begin{abstract}
Background: The aim of the study was to assess the morbidity and efficacy of repeat cryoablation (CA) in the treatment of localized prostate cancer.

Methods: Twenty-seven patients with median age of 71 years (range 48-80) who underwent repeat CA between April 2003 and April 2011 at a single institution were included. The median initial prostate-specific antigens (PSA) and Gleason values were $6.2 \mathrm{ng} / \mathrm{ml}$ (range 4-23.6) and 7 (range 6-9), respectively. Twenty-four patients underwent two CA treatments, and three patients underwent three CA treatments. Pre- and perioperative parameters and oncological and functional outcomes were evaluated.

Results: No intraoperative complications occurred. After the first CA, PSA was undetectable in 10 patients, and the median nadir PSA value was $0.65 \mathrm{ng} / \mathrm{ml}$ (range 0.1-4.9). After the second CA, 4 patients had undetectable PSA, and the median nadir PSA value was $1.25 \mathrm{ng} / \mathrm{ml}$ (range 0.2-7.9). For patients who underwent a third CA treatment, no patients had undetectable PSA, and the subsequent median nadir PSA value was $1.6 \mathrm{ng} / \mathrm{ml}$ (range 0.4-4.5). Two patients had incontinence ( 1 pad per day) following repeat CA. One patient had urinary retention after the third CA treatment, and one had urethral stricture. The mean hospitalization and follow-up periods were 1 day (range 0-2) and 51.5 months (range 11-96), respectively.

Conclusions: Repeat CA successfully reduced PSA levels, and complications were modest. We conclude that repeat CA is a feasible, safe, and effective treatment option for localized prostate cancer.
\end{abstract}

Keywords: Cryoablation, Prostate, Prostate cancer, Repeat cryoablation

\section{Background}

With the introduction of prostate-specific antigen (PSA) screening, an increasing number of men are being diagnosed with low-grade, low-stage, and small-volume cancers that are potentially biologically indolent. Choosing whether and how to treat such tumors remains challenging. Men newly diagnosed with low-risk prostate cancer are frequently treated with standard therapies, i.e., radical prostatectomy (RP), external beam radiation therapy, brachytherapy, androgen deprivation therapy, or conservative management [1]. These therapies are associated

\footnotetext{
* Correspondence: dr_mahmoud681@yahoo.com

${ }^{1}$ Urology Department, Faculty of Medicine and Health Science, An-Najah National University, An-Najah University Hospital, Nablus, West bank, Palestine

${ }^{2}$ Urology Department, MD Anderson Cancer Center, University of Texas, Houston, TX, USA
}

with high overall cancer-specific and biochemical recurrence-free survival; however, RP, radiation therapy, and androgen deprivation therapy are accompanied by side effects that may negatively affect patients' healthrelated quality of life. Conservative management was reported to induce anxiety and elevate stress: however, active surveillance for low-risk prostate cancer was strongly supported by Klotz et al. in many recent studies $[2,3]$. As such, renewed interest has emerged in using minimally invasive approaches such as CA to treat clinically localized prostate cancer.

Cryotherapy has become more widespread in practice because of a better understanding of cryobiology [4], introduction of third-generation cryoprobes, and improvement in biopsy and imaging techniques that have enhanced the ability to map the foci and 
locations of tumors within the prostate, subsequently reducing morbidity while improving effectiveness [4-6]. Information is lacking on whether $\mathrm{CA}$ is effective in the repeat setting when disease recurs after initial $\mathrm{CA}$, and there is no formal definition of CA eligible tumor. Moreover, morbidity associated with $\mathrm{CA}$ has been primarily reported from single hospital-based studies, typically in highly selected patients [7-13]. Herein, we are the first to report on the morbidity and efficacy of repeat CA as a primary and salvage option for the treatment of clinically localized prostate cancer.

\section{Methods}

We retrospectively evaluated the hospital records of patients who underwent a repeat $C A$ procedure at a single institution between April 2003 and April 2011. Twentyseven patients with median age 71 years (range 48-80) underwent repeat CA for biopsy-proven prostate cancer. Repeat cryotherapy procedures were performed with similar methodology to the initial salvage cryotherapy procedure which has been described in detail elsewhere. [14]. All procedures were performed under general anesthesia with the urethral warming catheter in place and set at a temperature of $42{ }^{\circ} \mathrm{C}$ to protect the urethra. Whole-gland CA was applied except in five patients where focal therapy was used. The median PSA value and Gleason score at initial diagnosis were $6.2 \mathrm{ng} / \mathrm{ml}$ (range 4-23.6) and 7 (range 6-9), respectively. The clinical stages were T1c in 15 patients, T2 in 11 patients, and $\mathrm{T} 3$ in 1 patient. The preoperative demographic characteristics and functional and oncological outcomes were summarized in Table 1. All patients had negative bone scan and computed tomography findings prior to application of CA. Staging transrectal ultrasonography was performed to rule out seminal vesicle involvement. Biopsy of the seminal vesicles is obtained at the time of transrectal ultrasoundguided prostate biopsies. After the prostate biopsies are obtained, the seminal vesicle tissue is visualized ultrasonographically, and two cores are obtained of each seminal vesicle. Fifteen patients (56\%) had undergone CA as primary therapy; however, 12 patients (44\%) had had hormone therapy (HT) and/or radiation therapy prior to first CA (Table 1). Neoadjuvant HT in 10 patients over a median period of 7 months (range 3-96). Twenty-four patients underwent two cryosurgical ablation procedures, and three patients had three procedures. One patient had the second CA for the right seminal vesicle involvement only, and one patient had open pelvic lymph nodes dissection (PLND) in conjunction with CA. Additional therapy was given for 13 patients: 8 received HT, 3 received $\mathrm{HT}$ and/or radiation therapy, 1 received radiation therapy, chemotherapy,
Table 1 Demographic characteristics and oncological and functional outcomes of the study group

\begin{tabular}{|c|c|}
\hline Variable & Value \\
\hline Patients $(n)$ & 27 \\
\hline Age (years, median, range) & $71(48-80)$ \\
\hline Initial PSA value (ng/ml, median, range) & $6.2(4-23.6)$ \\
\hline Initial volume of prostate before CA (cc, median, range) & $29(15-53)$ \\
\hline Volume of prostate after repeat CA (cc, median, range) & $18(10-48)$ \\
\hline \multicolumn{2}{|l|}{ Gleason score at initial diagnosis } \\
\hline $6(n \%)$ & $5(19)$ \\
\hline $7(n \%)$ & $13(48)$ \\
\hline 8 or $9(n \%)$ & $9(33)$ \\
\hline \multicolumn{2}{|l|}{ Initial clinical stage before first CA } \\
\hline $\operatorname{T1c}(n \%)$ & $15(55)$ \\
\hline $\mathrm{T} 2(n \%)$ & $11(41)$ \\
\hline T3 ( $n$ \%) & $1(4)$ \\
\hline \multicolumn{2}{|l|}{ Initial therapy before first CA } \\
\hline $\mathrm{HT}(n \%)$ & $6(22)$ \\
\hline HT and either RT or BT ( $n$ \%) & $5(19)$ \\
\hline $\mathrm{RT}(n \%)$ & $1(4)$ \\
\hline $\begin{array}{l}\text { Interval between first CA and positive biopsy } \\
\text { (median, months) }\end{array}$ & $23(6-63)$ \\
\hline PSA value at positive biopsy after first CA (median, $\mathrm{ng} / \mathrm{ml}$ ) & $2.7(0.2-7.8)$ \\
\hline \multicolumn{2}{|l|}{ Postoperative complications of repeat CA ( $n$ \%) } \\
\hline Continence (no pad usage) & $24 / 26(92)$ \\
\hline Stricture & $1 / 26(3.84)$ \\
\hline \multicolumn{2}{|l|}{ PSA value at last follow-up ${ }^{a}$} \\
\hline $\begin{array}{l}\text { Median value among patients with detectable PSA } \\
(\mathrm{ng} / \mathrm{ml})\end{array}$ & $0.8(0.2-50)$ \\
\hline Patients with undetectable PSA ( $n \%$ ) & $5 / 26^{\mathrm{a}}(19)$ \\
\hline Patients with PSA value $<0.5 \mathrm{ng} / \mathrm{ml}(n \%)$ & $13 / 26(50)$ \\
\hline Patients with PSA value $\geq 0.5 \mathrm{ng} / \mathrm{ml}(n \%)$ & $8 / 26(31 \%)$ \\
\hline
\end{tabular}

$n$ number of patients, CA cryoablation, PSA prostate-specific antigen, $H T$ hormonal therapy, $R T$ radiation therapy, $B T$ brachytherapy

${ }^{a}$ One patient did not return for follow-up after the second CA

and ketoconazole, and 1 patient underwent robotic RP. Ethical approval from MD Anderson Cancer Center was obtained, and the study was carried out in compliance with the Helsinki Declaration. The patients were informed about the operation and consent was obtained. Biochemical recurrence after CA was defined as detectable measurement of PSA. Continence was defined as use of no pads or a small liner for security purposes only. Potency was defined as the ability to achieve an erection adequate for penetration with or without medication (i.e., phosphodiesterase-5 inhibitors, intracavernous injection, or vacuum). The median hospitalization and follow-up periods were 1 day (range $0-2$ ) and 40.5 months (range 11-96). 


\section{Results}

The procedure was well tolerated in all patients, and no intraoperative complications occurred. The PSA value before the first CA procedure was $5.6 \mathrm{ng} / \mathrm{ml}$ (range 015). Ten patients (37\%) had undetectable PSA after the first CA. Clinical variables assessed before and after each CA procedure are summarized in Table 2. One patient did not return for follow-up after the second CA. The median nadir PSA values after the first, second, and third CA were $0.65,1.25$, and $1.6 \mathrm{ng} / \mathrm{ml}$, respectively. The median interval from first CA to first positive biopsy was 23 months (range 6-63), with median value of PSA $2.7 \mathrm{ng} / \mathrm{ml}$ (range 0.2-7.9). The median period between the first CA and the second CA was 27 months (range 7-78). The positive biopsy cores were located at the apex and/or base of the prostate in $65 \%$ of patients undergoing the first $\mathrm{CA}, 70 \%$ of patients undergoing the second CA, and $100 \%$ of patients undergoing a third CA. Only one patient had failure of HT and chemotherapies and developed bone metastasis 14 months after the first CA. Five patients $(5 / 26,19 \%$ ) (two of them without additional therapy) had undetectable PSA level at the last follow-up, and 8 patients $(8 / 26,31 \%)$ had detectable PSA values less than $0.5 \mathrm{ng} / \mathrm{ml}$. Twenty patients $(20 / 26.77 \%)$ had PSA values less than $2 \mathrm{ng} / \mathrm{ml}$ at the last follow-up, and the median PSA value was $0.8 \mathrm{ng} / \mathrm{ml}$ (range $0.2-50$ ) in all patients with detectable PSA.

According to Clavien classification system, postoperative complications were seen in five patients $(5 / 26$, $19 \%$ ); three patients had grade I, one patient had grade II, and one patient had grade IIIb. One patient (4\%) developed a severe lower urinary tract symptom of acute retention, which started as irritative symptoms after the first $\mathrm{CA}$ and increased after the second and third CA; injury of the prostatic urethra was observed after the third CA. One patient (4\%) had urethral stricture with overactive bladder symptoms, and a transurethral bladder neck resection was performed. Three patients (11\%) developed mild perioperative complications-mild lower urinary tract symptoms, scrotal edema, and perineal abscess which resolved spontaneously. One patient developed neuropathy in the lower extremity and spinal stenosis due to radiation therapy that was given after the third CA. Two patients $(8 \%)$ had incontinence with the need for one pad per day (Table 1). Those two patients were primary cases without history of radiotherapy before CA. One of them had history of urethral stricture and underwent transurethral resection. Regarding erectile dysfunction, only 6 patients were potent before CA, and 21 patients either had no available data about erectile dysfunction or could not receive medication for it due to older age or comorbid diseases. However, 5 patients $(19 \%)$ could achieve erection with medication (3 with intracavernous injection, 1 with phosphodiesterase5 (PDE-5) inhibitors, and 1 with a vacuum device) at the end of the second CA.

\section{Discussion}

Albeit selection criteria for men undergoing prostate CA have yet to be definitely established, optimal candidates for this procedure generally include those with lowerstage, lower-volume disease, and PSA values less than $20 \mathrm{ng} / \mathrm{ml}$ [15]. Our findings demonstrate the validity of $\mathrm{CA}$ as an effective and safe treatment option not only for lower-risk patients but also for the intermediate- or even high-risk patients with localized prostate cancer. Similarly, Roberts et al. reported, in an analysis of a Medicare-linked database from 2004 to 2005, that $16.1 \%$ of those who underwent CA therapy had highrisk disease [16]. In our series, only 5 patients (19\%)

Table 2 Pre- and postoperative clinical variables by cryoablation procedure

\begin{tabular}{|c|c|c|c|}
\hline Variable & First CA & Second CA & Third CA \\
\hline Patients $(n)$ & 27 & 27 & 3 \\
\hline Median PSA before CA (ng/ml, range) & $5.6(0-15)$ & $3(0-6.94)$ & $2(1.1-12.8)$ \\
\hline Median total cores at biopsy before CA ( $n$, range) & $10(4-14)$ & $12(6-92)$ & $13(12-60)$ \\
\hline Median positive cores at biopsy before CA ( $n$, range) & $3(1-12)$ & $2(1-9)$ & $4(1-7)$ \\
\hline Median percentage of positive cores before CA (\%, range) & $33(8-100)$ & $10(1-75)$ & $8(5-58)$ \\
\hline Median cumulative length of cancer at biopsy before CA ( $\mathrm{mm}$ ) & $16(1-136)$ & $4.5(1-53.5)$ & $17(1-31)$ \\
\hline Location of cancer at apex and/or base ( $n \%)$ & $15 / 23(65)$ & 19/27 (70) & $3 / 3(100)$ \\
\hline Location of cancer at apex ( $n$ \%) & 6/23 (26) & $12 / 27(44)$ & $1 / 3(33)$ \\
\hline Location of cancer at base $(n \%)$ & $3 / 23(13)$ & 4/27 (15) & $1 / 3(33)$ \\
\hline Undetectable PSA after CA ( $n$ \%) & 10/27 (37.03) & $4 / 27(14.81)$ & 0 \\
\hline Nadir PSA after CA (ng/ml, range) & $0.65(0.1-4.9)$ & $1.25(0.2-7.9)$ & $1.6(0.4-4.5)$ \\
\hline Duration of CA procedure (minutes) & $115(186-169)$ & $100(59-168)$ & $130(109-250)^{a}$ \\
\hline
\end{tabular}

$n$ number, CA cryoablation, PSA prostate-specific antigen

${ }^{a}$ One patient underwent open PLND with third CA 
had a Gleason score $<6$ and 9 patients (33\%) had a Gleason score of either 8 or 9 . The clinical stages for 5 patients (4 patients T2b, 1 patient T3) were also not confined to a low-risk group. One patient developed bone metastasis, $19 \%$ of the patients had undetectable PSA level, and $50 \%$ had a PSA level less than $0.5 \mathrm{ng} / \mathrm{ml}$ after CA. The patient who developed bone metastasis had a clinical stage of T2a, $100 \%$ of cores positive, and a high volume $(136 \mathrm{~mm})$ of cancer at biopsy with a Gleason score of 8 and 5 years of neoadjuvant hormone therapy; thus, this patient had a poor prognosis and benefit from other kinds of therapies was unlikely. Three patients had progressive disease from T1c to T3 (seminal vesicle involvement) after $C A ; 1$ patient underwent a second $C A$ for the right seminal vesicles, and his last PSA test yielded an undetectable level, and the other patient, who had a history of radiation therapy before $\mathrm{CA}$, underwent robotic $\mathrm{RP}$ without complication and at his last follow-up was under watchful waiting with a PSA level of $0.4 \mathrm{ng} / \mathrm{ml}$. One patient had a positive lymph node after the second CA and underwent open pelvic lymph node dissection. A biopsy after the last CA detected no cancer, and his PSA value at last follow-up $<0.1 \mathrm{ng} / \mathrm{ml}$. An advantage of CA for intermediate- or even high-risk patients with localized prostate cancer is that it does not hinder patients from undergoing other kinds of salvage therapies such as robotic RP; Pisters et al. reported on the feasibility of RP after neoadjuvant CA [17].

Cryotherapy can be used as a salvage treatment, and it is currently the only repeatable therapy for localized prostate cancer. Among salvage therapies, salvage RP and salvage CA represent the most feasible and effective therapeutic options for prostate cancer that recurs after radiation therapy. Salvage cryotherapy is performed four times as often as salvage RP, although both procedures remain underutilized for the management of recurrent prostate cancer $[18,19]$. Pisters et al. performed a stratified control comparison of biochemical disease-free survival after salvage RP and salvage CA [20]. Compared to salvage CA, salvage RP resulted in superior biochemical disease-free survival, $21 \%$ for salvage CA vs $61 \%$ for salvage RP at 5 years [20]. Thus, salvage CA is best considered in older patients who decline to undergo salvage RP. However, there is no curative therapy in prostate cancer that is not responding to radiotherapy, and there is no definition of success for CA. The PSA level before salvage $\mathrm{CA}$ and Gleason score correlated with biochemical progression-free survival (bPFS): the initial PSA level of $<0.6 \mathrm{ng} / \mathrm{mL}$ after salvage CA portends a favorable (67 \% at 36 months) bPFS [21]. In our series, CA was used as salvage therapy for 12 patients (44 \%, 6 of whom had previous radiation therapy (RT)) and as primary therapy for 15 patients. Although the previously reported intraoperative and postoperative complication rates have been high after salvage CA, our patients had no rectal injury, bowel bleeding, or hydronephrosis. Therefore, CA is not associated with the high intraoperative complication rate of other kinds of salvage therapies, and it is a feasible treatment modality in patients for whom initial therapies, including radiation and CA, have failed. Many recent studies have reported on the validity of salvage CA ablation after radiation therapy for prostate cancer [22]; however, we found only one study about the feasibility of repeat CA after the failure of the primary CA [23]. In one of these studies, de Castro and his colleagues compared salvage focal with standard salvage total CA for locally recurrent prostate cancer after primary radiation therapy [22]. They concluded that salvage focal and salvage total CA are feasible and safe with acceptable mid-term oncological outcomes and that morbidity was lower for salvage focal CA than for salvage total CA [22]. Focal therapy is a modification of the standard CA technique aiming to only treat the portion of the prostate gland which has the cancer with minimization of complications especially potency. This kind of therapy was introduced by Onik et al. [24] in 2004 and supported by many recent studies regarding its efficacy in the treatment of primary and radiorecurrent locally prostate cancer [25].

The postoperative complications occurring after CA have been widely studied in small single hospital-based studies. Urinary incontinence ranged from 1.3 to $9.5 \%$ $[9-11,13,26]$, urinary strictures from 1.7 to $17 \%[10,13]$, lower urinary tract obstruction and retention from 6.6 to $13 \%$ [25], erectile dysfunction from 47 to $94.9 \%$ $[10,11,13]$, and fistula less than $1 \%[10-13]$. In our cohort of men receiving repeat $\mathrm{CA}$, the majority of the postoperative complications were clinically insignificant. According to Clavien classification system, only one patient had grade IIIB complication and endoscopic management was done. No urinary or bowel fistula was observed. Incontinence occurred in two patients (8\%), with only one pad per day. One of them had had rectal abscess surgery 24 years before $\mathrm{CA}$, and he suffered from mild nocturia after the first $\mathrm{CA}$, which increased after the second CA. The second patient had urethral stricture with overactive bladder symptoms after the second CA; thus, bladder neck transurethral resection was done 32 months after the second CA. This patient had lower urinary tract symptoms before the first CA and had a history of urethral stricture after a fall from a tree; several urethral dilations had been done before the first CA. Thus, postoperative complications occurring after CA were not only attributable to the CA but also to the previous history. Another complication seen (one patient) was severe lower urinary tract symptoms and urinary retention after the third CA, due to injury of the prostatic urethra; thus, a Foley catheter was placed. This patient also had a history 
of irritative symptoms, which increased after the first CA and became severe after the third CA. Erectile dysfunction is a well-known side effect of CA; in our study, although the patients were older and the majority of the patients either were not potent before CA or were not interested in sex therapy, five patients could achieve erection with the help of medication after the last CA. The evaluation of erectile dysfunction in our study was suboptimal; therefore, it is difficult to make a definitive comment regarding potency. However, $19 \%$ of the patients after repeat CA could achieve erections sufficient for penetration, which is a promising finding.

Location of cancer in the prostate base and/or apex is one of the greatest challenges for application of CA. The use of thermocouples in the apex and base to document adequate freezing temperatures could improve the results of salvage cryotherapy. In particular, the apex is sometimes difficult to visualize ultrasonographically and a thermocouple in both the right and left apical tissue may improve the effectiveness of salvage cryotherapy. In our study, the proportions of patients with cancer sited at the base and/or apex before the first, second, and third treatments were 65,70 , and $100 \%$, respectively (Table 2). Two patients who achieved undetectable PSA levels after the last CA had such cancers (one patient, cancer at the apex; and one patient, cancer at the base and apex). An undetectable PSA level is unlikely to be achieved after CA because a rim of PSA-producing periurethral tissue will invariably remain. Biopsy is the most definitive determination of failure or success; however, there is no consensus on when and at which PSA level should be performed.

\section{Conclusions}

Our study demonstrates that repeat CA is both safe and effective. Cryotherapy as a primary or salvage option can be repeated with a modest complication rate and reasonable efficacy. Low- and high-risk localized prostate cancer can be treated with CA without hindering the patients from undergoing other kinds of salvage therapies. Base or/ and apex of the prostate are the most common place for the recurrence after $\mathrm{CA}$.

\section{Competing interests}

The authors declare that they have no competing interests.

\section{Authors' contributions}

MM contributed to the protocol development, data collection, data analysis, and writing. SD contributed to the data collection. LP contributed to the protocol development and manuscript editing. All authors read and approved the final manuscript.

\section{Informed consent}

The patients were informed about the operation and consent was obtained from the patients. The study was carried out under the protocol number of PA11-0402.
Researches involving Human Participant and/Animals

Certificate from National Institute of health (NIH), Number 1720774.

Received: 19 September 2015 Accepted: 18 December 2015

Published online: 21 December 2015

\section{References}

1. Cooperberg MR, Lubeck DP, Meng MV, Mehta SS, Carroll PR. The changing face of low-risk prostate cancer: trends in clinical presentation and primary management. J Clin Oncol. 2004;22:2141-9.

2. Dale W, Bilir P, Han M, Meltzer D. The role of anxiety in prostate carcinoma: a structured review of the literature. Cancer. 2005;104:467-78.

3. Klotz L, Vesprini $D$, Sethukavalan $P$, Jethava $V$, Zhang $L$, Jain $S$, et al. Long-term follow-up of a large active surveillance cohort of patients with prostate cancer. J Clin Oncol. 2015;33(3):272-7.

4. Babaian RJ, Donnelly B, Bahn D, Baust JG, Dineen M, Ellis D, et al. Best practice statement on cryosurgery for the treatment of localized prostate cancer. J Urol. 2008;180:1993-2004.

5. Saliken JC, Donnelly BJ, Rewcastle JC. The evolution and state of modern technology for prostate cryosurgery. Urology. 2002;60:26-33.

6. Sartor AO, Hricak H, Wheeler TM, Coleman J, Penson DF, Carroll PR, et al. Evaluating localized prostate cancer and identifying candidates for focal therapy. Urology. 2008;72(6 Suppl):S12-24.

7. Malcolm JB, Fabrizio MD, Barone BB, Given RS, Lance DF, Lynch JW, et al. Quality of life after open or robotic prostatectomy, cryoablation or brachytherapy for localized prostate cancer. J Urol. 2010;183:1822-8.

8. Ellis DS, Manny Jr TB, Rewcastle JC. Focal cryosurgery followed by penile rehabilitation as primary treatment for localized prostate cancer: initial results. Urology. 2007;70:9-15.

9. Donnelly BJ, Saliken JC, Ernst DS, Ali-Ridha N, Brasher PM, Robinson JW, et al. Prospective trial of cryosurgical ablation of the prostate: five-year results. Urology. 2002;60:645-9.

10. Aus G, Pileblad E, Hugosson J. Cryosurgical ablation of the prostate: 5-year follow-up of a prospective study. Eur Urol. 2002:42:133-8.

11. Bahn DK, Lee F, Badalament R, Kumar A, Greski J, Chernick M. Targeted cryoablation of the prostate: 7-year outcomes in the primary treatment of prostate cancer. Urology. 2002;60:3-11.

12. Prepelica KL, Okeke Z, Murphy A, Katz AE. Cryosurgical ablation of the prostate: high risk patient outcomes. Cancer. 2005;103:1625-30.

13. Long JP, Fallick ML, LaRock DR, Rand W. Preliminary outcomes following cryosurgical ablation of the prostate in patients with clinically localized prostate carcinoma. J Urol. 1998;159:477-84.

14. Ward JF, Pagliaro LC, Pisters LL. Salvage therapy for radiorecurrent prostate cancer. Curr Probl Cancer. 2008;32(6):242-71

15. Finley DS, Pouliot F, Miller DC, Belldegrun AS. Primary and salvage cryotherapy for prostate cancer. Urol Clin North Am. 2010;37:67-82.

16. Roberts CB, Jang TL, Yu-Hsuan S, Kabadi S, Moore DF, Lu-Yao GL. Treatment profile and complications associated with cryotherapy for localized prostate cancer: a population-based study. Prostate Cancer Prostatic Dis. 2011;14:313-9.

17. Plsters LL, Dinney CP, Pettaway CA, Scott SM, Babaian RJ, von Eschenbach $A C$, et al. Feasibility study of cryotherapy followed by radical prostatectomy for locally advanced prostate cancer. J Urol. 1999;161:509-14.

18. Pisters LL. Treatment failure after primary and salvage therapy for prostate cancer. Cancer. 2008;112:225-7.

19. Agarwal PK, Sadetsky N, Konety BR, Resnick MI, Carroll PR, Cancer of the Prostate Strategic Urological Research Endeavor (CaPSURE). Treatment failure after primary and salvage therapy for prostate cancer: likelihood, patterns of care, and outcomes. Cancer. 2008;112:307-14.

20. Pisters LL, Leibovici D, Blute M, Zincke H, Sebo TJ, Slezak JM, et al. Locally recurrent prostate cancer after initial radiation therapy: a comparison of salvage radical prostatectomy versus cryotherapy. J Urol. 2009;182:517-25.

21. Levy DA, Pisters LL, Jones JS. Prognostic value of initial prostate-specific antigen levels after salvage cryoablation for prostate cancer. BJU Int. 2010; 106(7):986-90.

22. de Castro Abreu AL, Bahn D, Leslie S, Shoji S, Silverman P, Desai MM, et al. Salvage focal and salvage total cryoablation for locally recurrent prostate cancer after primary radiation therapy. BJU Int. 2013;112:298-307.

23. Chang X, Liu T, Zhang F, Zhao X, Ji C, Yang R, et al. Salvage cryosurgery for locally recurrent prostate cancer after primary cryotherapy. Int Urol Nephrol. 2015;47(2):301-5. 
24. Onik G. The male lumpectomy: rationale for a cancer targeted approach for prostate cryoablation. A review. Technol Cancer Res Treat. 2004;3:365-70.

25. Li YH, Elshafei A, Agarwal G, Ruckle H, Powsang J, Jones JS. Salvage focal prostate cryoablation for locally recurrent prostate cancer after radiotherapy: initial results from the cryo on-line data registry. Prostate. 2015;75:1-7.

26. Kimura M, Mouraviev V, Tsivian M, Moreira DM, Mayes JM, Polascik TJ.

Analysis of urinary function using validated instruments and uroflowmetry after primary and salvage prostate cryoablation. Urology. 2010;76:1258-65.

Submit your next manuscript to BioMed Central and we will help you at every step:

- We accept pre-submission inquiries

- Our selector tool helps you to find the most relevant journal

- We provide round the clock customer support

- Convenient online submission

- Thorough peer review

- Inclusion in PubMed and all major indexing services

- Maximum visibility for your research

Submit your manuscript at www.biomedcentral.com/submit 